\title{
Phantom validation of 17 and 11 heartbeat MOLLI T1 mapping sequence at 3T
}

Songtao Liu ${ }^{1 *}$, Justin Huang ${ }^{1}$, Martin Ugander ${ }^{2}$, Christopher Sibley ${ }^{1}$, Abiola Oki ${ }^{2}$, Jing Han ${ }^{3}$, Andreas Greiser ${ }^{4}$, Daniel R Messroghli ${ }^{5}$, Peter Kellman², Andrew E Arai ${ }^{2}$, David A Bluemke ${ }^{1}$

From 2011 SCMR/Euro CMR Joint Scientific Sessions

Nice, France. 3-6 February 2011

\section{Background and objective}

The Modified Look-Locker Inversion-Recovery (MOLLI) sequence (Messroghli, et al, JMRI, 2007) was optimized for myocardial T1 mapping on 1.5T. However, little data exists on T1 mapping at 3T. The standard MOLLI sequence uses three inversion-recovery blocks to acquire 11 images over 17 heartbeats (HB). The long breath hold could limit its clinical application in patients with cardiorespiratory compromise. A new 11 HB MOLLI protocol with two inversion-recovery blocks was introduced recently, which reduces scan time by $35 \%$. This aim of this study is to verify both $17 \mathrm{HB}$ and $11 \mathrm{HB}$ MOLLI sequences at 3T.

\section{Methods}

Agarose gel-cupric sulfate phantoms were scanned using both $17 \mathrm{HB}$ and $11 \mathrm{HB}$ MOLLI sequence at 3T (Verio, Siemens) with simulated heart rates from $40-110$ beats per minute (bpm). An inversion-recovery spin-echo (IR-SE) sequence with TR of $10 \mathrm{sec}$, TI varying from $10 \mathrm{~ms}$ to $2500 \mathrm{~ms}$ was used in the same configuration. The phantoms were also scanned using a spin-echo

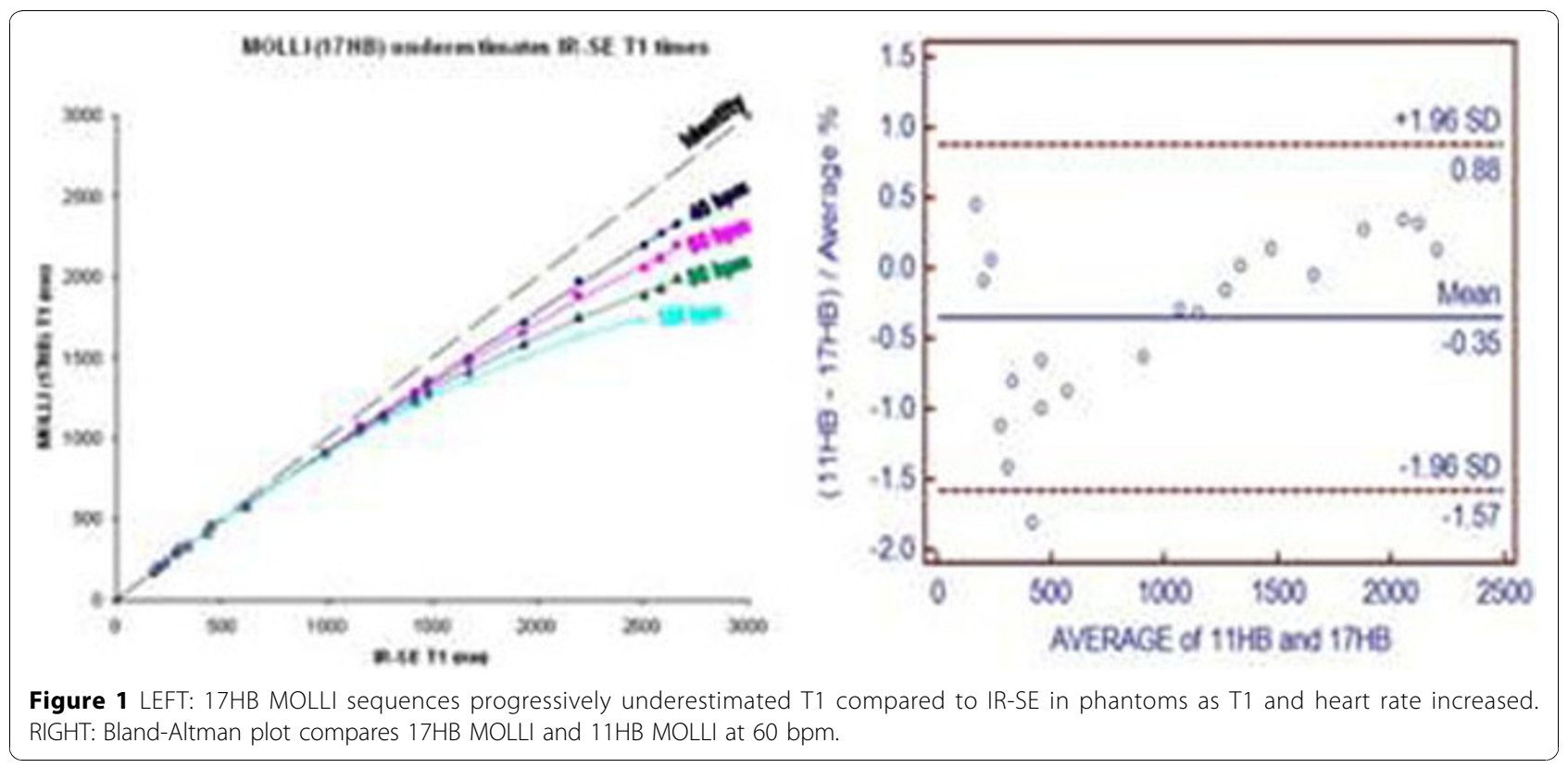

${ }^{1} \mathrm{NIH}$ Clinical Center, Bethesda, MD, USA

Full list of author information is available at the end of the article 
sequence with varying TE for T2 quantification. T1 maps were generated by MRMap (Messroghli, BMC Medical Imaging, 2010) and a region of interest inside each phantom was measured by ImageJ. Results were compared to each other and to IR-SE, which served as the reference standard. The phantoms studied had T1 times that ranged from $174-2660 \mathrm{~ms}$ and T2 times ranging from $51-188 \mathrm{~ms}$

\section{Results}

T1 estimates by both 17HB and 11HB MOLLI matched measurements by IR-SE up to $\sim 500 \mathrm{~ms}$, but underestimated longer T1 times. This underestimation increased for higher heart rates (see figure). After correction, the difference between 17HB MOLLI and IR-SE was reduced to $-0.8+/-2.3 \%$, and difference between 11HB MOLLI and IR-SE was $-0.4 \%+/-2.2 \%$. The mean difference between $17 \mathrm{HB}$ MOLLI and $11 \mathrm{HB}$ MOLLI was $0.4+/-1.2 \%$ at $60 \mathrm{bpm}$, and this was similar at other heart rates.

\section{Conclusions}

T1 mapping by MOLLI is feasible and accurate at 3T. Calibrated heart rate correction is necessary for $\mathrm{T} 1$ times longer than $500 \mathrm{~ms}$. Within our sample, $11 \mathrm{HB}$ MOLLI was an equally accurate estimator of IR-SE compared to 17HB MOLLI after correction was applied. Based on this, 17HB MOLLI and 11HB MOLLI can be used interchangeably.

\section{Author details}

${ }^{1} \mathrm{NIH}$ Clinical Center, Bethesda, MD, USA. ${ }^{2} \mathrm{NHLBI}$, Bethesda, MD, USA. ${ }^{3}$ FDA Rockville, MD, USA. ${ }^{4}$ Siemens AG, Erlangen, Germany. ${ }^{5}$ Franz-Volhard-Klinik Charité, Berlin, Germany.

Published: 2 February 2011
Submit your next manuscript to BioMed Central and take full advantage of:

- Convenient online submission

- Thorough peer review

- No space constraints or color figure charges

- Immediate publication on acceptance

- Inclusion in PubMed, CAS, Scopus and Google Scholar

- Research which is freely available for redistribution 